\title{
Visuo-motor related time analysis using electroencephalograms*
}

\author{
Kengo Yotani ${ }^{1 \#}$, Hiroyuki Tamaki ${ }^{2}$, Hiroki Nakamoto ${ }^{1}$, Atsumu Yuki ${ }^{3}$, Hikari Kirimoto ${ }^{2}$, Koji Kitada ${ }^{4}$, \\ Futoshi Ogita $^{1}$, Shiro Mori ${ }^{1}$
}

${ }^{1}$ National Institute of Fitness and Sports in Kanoya, Kagoshima, Japan

${ }^{2}$ Niigata University of Health and Welfare, Niigata, Japan

${ }^{3}$ National Center for Geriatrics and Gerontology, Obu, Japan

${ }^{4}$ Ishikawa National College of Technology, Ishikawa, Japan

Email: "\#otani@nifs-k.ac.jp

Received 25 April 2013; revised 2 June 2013; accepted 5 July 2013

Copyright (C) 2013 Kengo Yotani et al. This is an open access article distributed under the Creative Commons Attribution License, which permits unrestricted use, distribution, and reproduction in any medium, provided the original work is properly cited.

\begin{abstract}
The objective of the present study was to assess the relationship of response time and peak latency of P300 with a simple-reaction task. Seven male subjects who were experienced players in decision-making sports were included. Two main findings were noted. First, the P300 latencies of $\mathrm{Fz}$ and $\mathrm{Pz}$ were correlated with visuo-motor related time. Second, in terms of latencies of visual evoked potentials, the correlations were observed only between Oz-P2 latency and visuo-motor related time. These results suggest that the length of visuo-motor related time is related to the processing involved in the higher order brain site involving specific processing in the visual cortex.
\end{abstract}

Keywords: Simple-Reaction Task; P300; Visual Evoked Potentials; Athletes

\section{INTRODUCTION}

In a simple-reaction task in which subjects respond as quickly as possible to a visual cue, pre-motor time (PMT) is measured as the delay between stimulus onset and onset of myoelectric activity using electromyography (EMG). A previous study reported that PMT can be subdivided into two factors: elapsed time from visual stimulation to the primary motor cortex (M1) through the visual cortex (visuo-motor related time [VMRT]), and from M1 to the muscle (motor evoked potentials [MEP] latency) [1]. Thus, VMRT is calculated as PMT minus MEP latency. The

\footnotetext{
*This work was supported in part by a Grant-in-Aid for Young Scientists (B) from the Japan Society for the Promotion of Science and from the National Institute of Fitness and Sports in Kanoya.

${ }^{*}$ Corresponding author.
}

VMRT is likely to reflect the time of visuo-motor integration and control [2]. VMRT differs in individuals [1]; however, the factors that define the length of time for VMRT are unclear.

Peak latency of P300 (P3) in event-related potentials is well recognized to be associated with reaction time; this has been supported by a number of studies using electroencephalograms (EEG) [3-7]. The P3 component links to cognitive processing of context updating in the particular higher order brain area [8-10], and peak latency is considered a measure of the processing speed via stimulus evaluation [11]. Given that P3 latency reflects the speed of central nervous processing, it seems useful to assess the length of VMRT. Moreover, VMRT, including processing of the visual system, requires consideration of the elapsed speed from visual stimulation to visual cortex activation.

The objective of the present study was to assess the relationship of response time and P3 latency with a simple-reaction task, that is, to examine the length of VMRT. In addition, we also investigated the latency in visual evoked potentials (VEP), that is, potentials indicating neural activities related to visual processing [12-14].

\section{MATERIAL AND METHODS}

Seven male subjects who were experienced players in decision-making sports, such as baseball, kendo, judo, basketball, or tennis, were included. All subjects had regularly attended local competitions for more than 8 years. Subjects had a mean age \pm standard deviation (SD) of $24.7 \pm 3.6$ years, height of $167.5 \pm 5.3 \mathrm{~cm}$, and weight of $64.1 \pm 9.1 \mathrm{~kg}$. Informed consent was obtained before beginning the experiment, which was conducted according to the Declaration of Helsinki. The experimental 
procedures were approved by the Ethics Committee of the National Institute of Fitness and Sports in Kanoya.

Subjects were comfortably seated in an experimental chair in an electrically shielded, sound-attenuated room. Their forearms rested on an armrest while their hands were kept in a neutral position. After a warning signal was presented, a visual signal was presented by a red light-emitting diode (LED) that was $1 \mathrm{~m}$ in front of subjects, at eye level. When the LED lit up, subjects were asked to contract the right flexor pollicis brevis muscle as quickly as possible in reaction to the visual signal and then to relax the muscle. The inter-stimulus interval was varied randomly from 2 to $6 \mathrm{sec}$ from the warning signal to avoid an anticipation effect for timing of the visual signal. Subjects were requested to avoid eye blinking during the task and to keep the right flexor pollicis brevis muscle relaxed between each trial. Complete muscle relaxation and eye movements were confirmed online via audiovisual feedback by EMG activity and an electrooculogram (EOG). The trials including eye blinks or other signal artifacts were excluded, and each subject performed the task until 50 artifact-free trials were obtained.

During the task, EEG was recorded with $\mathrm{Ag} / \mathrm{AgCl}$ electrodes from $\mathrm{Fz}, \mathrm{Cz}, \mathrm{Pz}$ and $\mathrm{Oz}$ according to the international $10-20$ system with reference to linked earlobes. An EOG was recorded using electrodes above and below the left eye. The EEG and EOG signal were sampled at $1000 \mathrm{~Hz}$, filtered with a low-pass frequency of $300 \mathrm{~Hz}$, and recordings were performed with a 32-channel digital DC EEG amplifier (NuAmps model; Compumedics NeuroScan, Charlotte, NC). The EMG activity of the right flexor pollicis brevis muscle was recorded using surface EMG electrodes. A DL-141 single-differential, parallelbar configuration (4 Assist, Inc., Japan) was put on the skin surface over the muscle. The EMG signal was filtered during acquisition with a bandwidth of 5 to $500 \mathrm{~Hz}$ and a gain of $\times 1000$. The signal was digitized at $1000 \mathrm{~Hz}$ (16 bit, PowerLab, AD Instruments, Japan), recorded, and stored for off-line analysis (Chart 6, AD Instruments) on a personal computer. The MEP elicited by transcranial magnetic stimulation (TMS) was recorded from the flexor pollicis brevis muscle at rest. TMS was performed using a figure-eight coil (outer diameter $70 \mathrm{~mm}$ ) connected to a monophasic Magstim 200 stimulator (Magstim, 200, UK). To define the optimal stimulation position (hot spot) for the muscle activation, the coil was placed over the left hemisphere, approximately $5 \mathrm{~cm}$ lateral to the $\mathrm{Cz}$ with the handle pointing backward and $45^{\circ}$ laterally from the middle line. The site at which TMS of a slightly suprathreshold intensity consistently elicited the largest MEP in the muscle was marked as the motor hotspot. TMS was defined as 1.1 to 1.2 times the intensity at which TMS evoked MEP at 50\% probability in 10 to 15 trials [15]. We provided the stimulus at 5 -sec intervals.

Response time was measured from the recorded light signal and EMG. PMT was defined as the period between the onset of visual stimulus and the first deflection of response activity noted on EMG (Figure 1A). The VMRT was calculated as PMT minus MEP latency (from TMS stimulus to onset of MEP; Figure 1B), which was identified as the period between the onset of visual signal and M1 [1]. The analysis epoch for stimulus-locked EEG was 1000 msec including a pre-stimulus (light) baseline period of $100 \mathrm{msec}$. Peak latency of $\mathrm{P} 3$ was measured at 250 - 500 msec from Fz, Cz, and Pz. VEP components (N1, P1, N2, and P2) were recorded from Oz, and the peak latency was measured at $50-100 \mathrm{msec}, 70-140$ msec, $100-190 \mathrm{msec}$ and $130-260 \mathrm{msec}$, respectively. All data were expressed as mean \pm SD (Table 1). Correlation analysis was conducted to evaluate relationship between P3, VEP latencies, and response time, especially in VMRT.

\section{RESULTS}

Table 2 shows the correlation coefficient analyses results between the response time and each of P3 and VEP latencies. Significant positive correlations were observed between Fz-, Pz-P3 latency and PMT, VMRT ( $\mathrm{r}=0.77$ $0.85, P<0.05$; Table 2 and Figure 2(a)), and no significant correlations were observed between Cz-P3 latency. In the VEP, significant negative correlations were observed only between Oz-P2 latency and PMT, VMRT ( $\mathrm{r}$ $=-0.76, P<0.05$; Table 2 and Figure 2(b)). There was no significant correlation between MEP latency and each of P3 and VEP latencies.

\section{DISCUSSION}

There were two main findings in the present study. First, the P3 latencies of $\mathrm{Fz}$ and $\mathrm{Pz}$ were correlated with VMRT. Second, in terms of latencies for VEP, correlations were observed only between the Oz-P2 latency and VMRT.

MEP latency typically includes a central motor conduction time and a peripheral motor conduction time. The variation of MEP latency is affected by age but not by athletic training $[1,16,17]$. Our results also did not show that latencies of P3 or VEP were correlated with MEP latency. For healthy coeval subjects, as in the present study, MEP latency appears to be independent of rapidity of the visual response. The P3 latency reflects a measure of stimulus classification or evaluation speed. Several authors have discussed P3 components in visual event-related potentials. It was reported that P3 consists of complex waves of the higher order brain site originating from the corpus callosum [18] and/or cortical communication system [19]. In addition, previous studies 
A

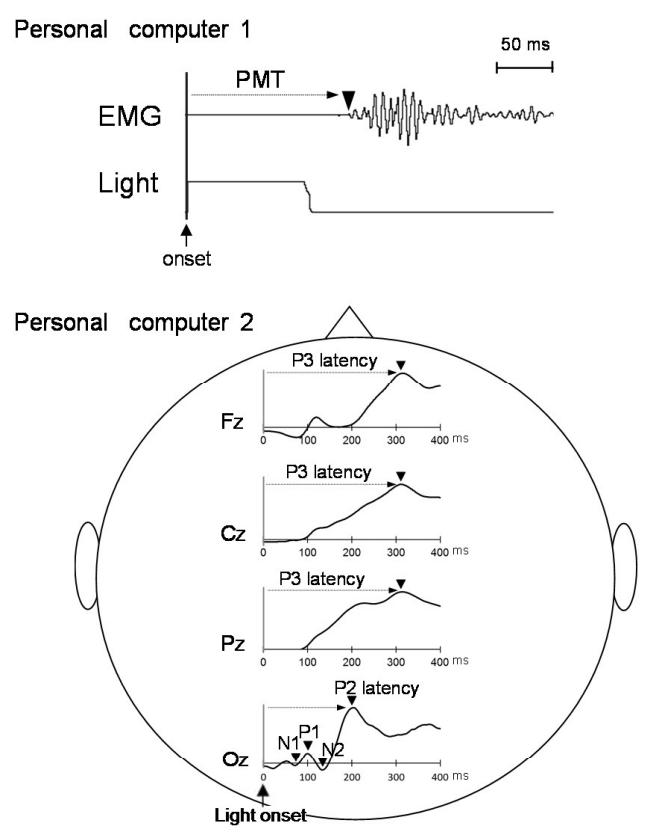

B
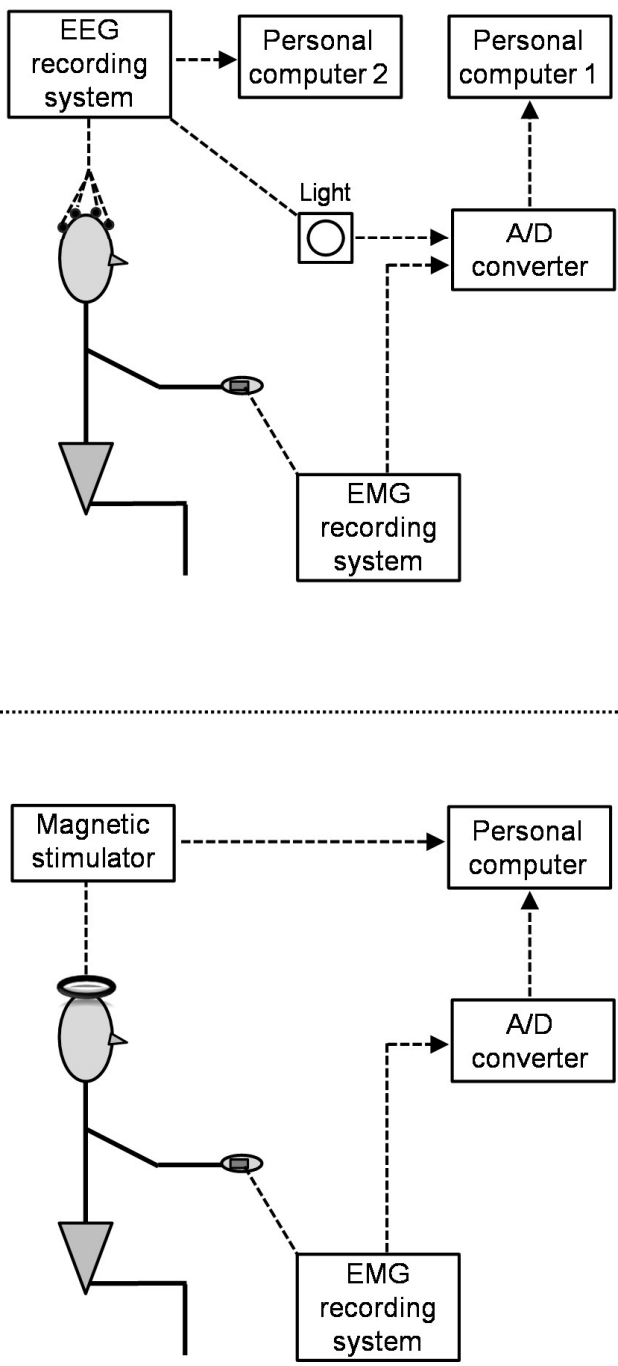

Personal computer

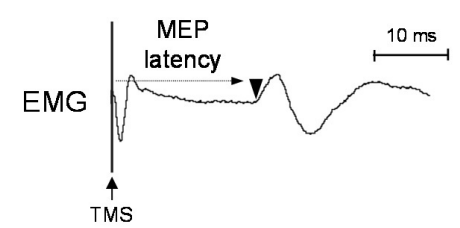

Figure 1. Original electromyography (EMG) and electroencephalograms (EEG), measured at the simple-reaction task (A) or transcranial magnetic stimulation TMS (B). PMT, pre-motor time; MEP, motor evoked potentials.

Table 1. Mean values and standard deviation of response time (pre-motor time [PMT], motor evoked potential [MEP] latency and visuo-motor related time [VMRT]) and peak latency of P300 (P3), visual evoked potentials (VEP).

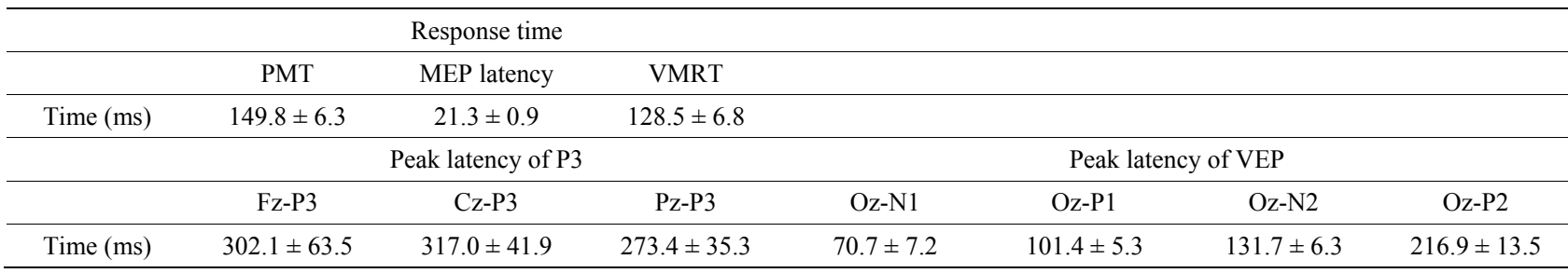

using TMS and functional magnetic resonance imaging have shown that the posterior parietal cortex and frontal cortex are concerned with visuo-motor integration [2] and attentional control [20]. Our results showed that VMRT was positively correlated with P3 latency on Fz and Pz.
Neural activities of the $\mathrm{Fz}$ and $\mathrm{Pz}$ regions might be the factors associated with the length of VMRT.

Our second finding demonstrated a negative correlation between Oz-P2 and VMRT. Possible mechanisms that explain this correlation may result from visual proc- 
Table 2. Correlation coefficients between response time parameters (pre-motor time [PMT], motor evoked potential [MEP] latency and visuo-motor related time [VMRT]) and peak latency of P300 (P3), visual evoked potentials (VEP).

\begin{tabular}{ccccc}
\hline & \multicolumn{3}{c}{ Response time } \\
\hline Peak latency of P3 & Fz-P3 & $0.875^{\mathrm{a}}$ & -0.337 & $0.845^{\mathrm{a}}$ \\
& Cz-P3 & -0.027 & 0.245 & -0.056 \\
& Pz-P3 & $0.797^{\mathrm{a}}$ & -0.264 & $0.771^{\mathrm{a}}$ \\
\hline Peak latency of VEP & Oz-N1 & 0.047 & 0.308 & 0.003 \\
& Oz-P1 & 0.177 & 0.496 & 0.101 \\
& Oz-N2 & -0.178 & 0.397 & -0.215 \\
& Oz-P2 & $-0.756^{\mathrm{a}}$ & 0.488 & $-0.760^{\mathrm{a}}$ \\
\hline
\end{tabular}

$$
{ }^{\mathrm{a}} P<0.05 \text {. }
$$
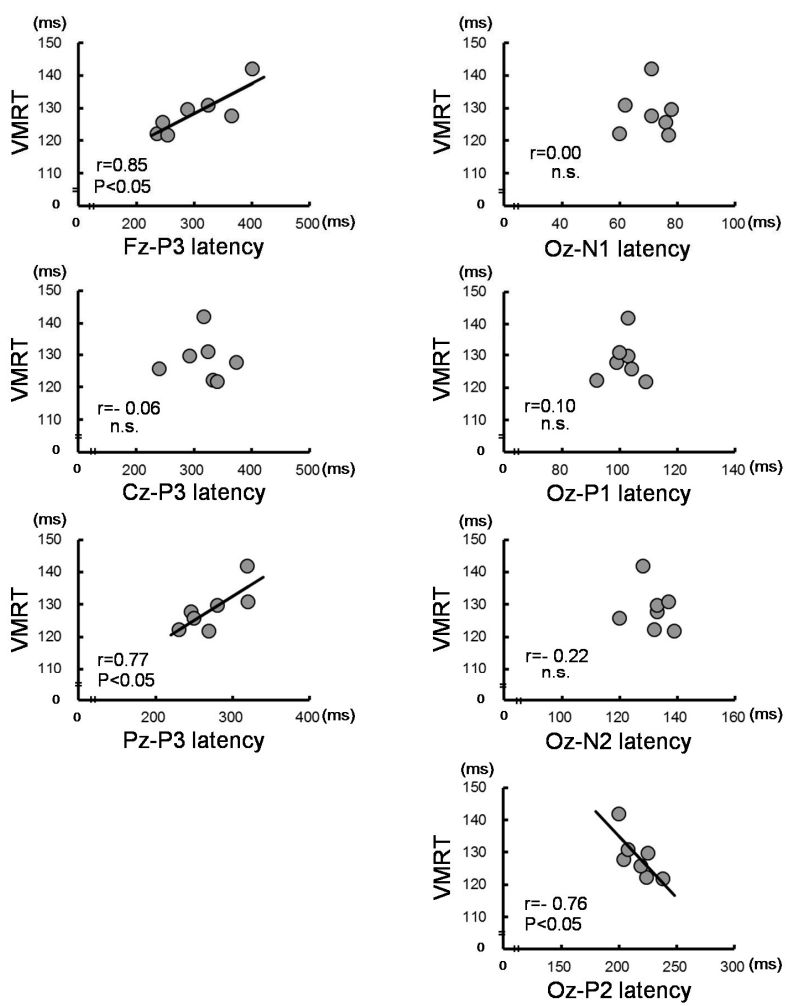

(a)

(b)

Figure 2. Correlation between visuo-motor related time (VMRT) and peak latency of P300 (P3) and visual evoked potentials (VEP). Significant correlations were observed between VMRT and Fz-, Pz-P3 latency (a), and Oz-P2 latency in VEP (b).

essing after primary visual cortex (V1) neurons. Because they are neuroanatomical connections, the extrastriate areas in the visual cortex, such as the secondary visual cortex (V2) and middle temporal (MT) neurons, receive information from the V1 neurons. These areas are not independent of each other but are mutually connected, and the frontal or parietal cortex receives information from the MT neurons in the end [21]. Previous brain studies of rats showed that neural circuits in the visual cortex have the ability to open a shortcut circuit with repetitive stimulation [22]. In addition, the P2 component in the VEP is known to be generated by current sources in the V2 neurons [23]. Our results showed that subjects who have longer P2 latency exhibit shorter VMRT (Figure 2(b)). However, even though subjects in this study also repeatedly conducted the same simple task, the idea of a shortcut circuit is unlikely to be explained based on our results. On the other hand, Raiguel et al. [24] reported that response latencies of visual cells after V1 neurons in the monkey are shorter in MT neurons than in V2 neurons. Considering the length of VMRT, which includes processing in the higher order brain, the negative correlation might represent a pattern that excited MT neurons more preferentially than V2 neurons after V1 neurons.

A principal limitation of this study is that results are based on a simple task. In addition, the relationship between VMRT and P3 or VEP latencies might have been affected by the fact that our subjects were athletes instead of sedentary people.

In conclusion, this study found that P3 or VEP latencies correlate with VMRT but not MEP latency in PMT. Our results suggest that the length of the VMRT is related to the processing involved in the higher order brain site involving specific processing in the visual cortex.

\section{REFERENCES}

[1] Yotani, K., Tamaki, H., Yuki, A., Kirimoto, H., Kitada, K., Ogita, F. and Takekura, H. (2011) Response training shortens visuo-motor related time in athletes. International Journal of Sports Medicine, 32, 586-590. doi:10.1055/s-0031-1275299

[2] Iacoboni, M. (2006) Visuo-motor integration and control in the human posterior parietal cortex: Evidence from TMS and fMRI. Neuropsychologia, 44, 2691-2699. doi:10.1016/j.neuropsychologia.2006.04.029

[3] Ford, J.M., Pfefferbaum, A. and Kopell, B.S. (1982) Effects of perceptual and cognitive difficulty on P3 and RT in young and older adults. Electroencephalography and Clinical Neurophysiology, 54, 311-321. doi:10.1016/0013-4694(82)90180-8

[4] Goodin, D.S. and Aminoff, M.J. (1984) The relationship between the evoked potential and brain events in sensory discrimination and motor response. Brain, 107, 241-251. doi:10.1093/brain/107.1.241

[5] Hohnsbein, J., Falkenstein, M., Hoormann, J. and Blanke, L. (1991) Effects of crossmodal divided attention on late ERP components. I. Simple and choice reaction tasks. Electroencephalography and Clinical Neurophysiology, 78, 438-446. doi:10.1016/0013-4694(91)90061-8

[6] Nishihira, Y., Yoshida, Y., Hatta, A., Kaneda, T., Kamijo, K., Higashiura, T., Kim, S.R., Yoshida, M. and Kim, B.J. (2005) Effects of task-responding manipulations on P300 and S-R compatibility. Advances in Exercise and Sports Physiology, 11, 119-124. 
[7] Duncan-Johnson, C.C. and Donchin, E. (1982) The P300 component of the event-related brain potential as an index of information processing. Biological Psychology, 14, 1-52. doi:10.1016/0301-0511(82)90016-3

[8] Bledowski, C., Prvulovic, D., Hoechstetter, K., Scherg, M., Wibral, M., Goebel, R. and Linden, D.E. (2004) Localizing P300 generators in visual target and distractor processing: A combined event-related potential and functional magnetic resonance imaging study. The Journal of Neuroscience, 24, 9353-9360. doi:10.1523/JNEUROSCI.1897-04.2004

[9] Donchin, E. and Coles, M.G. (1988) Is the P300 component a manifestation of context updating? Behavioral and Brain Sciences, 11, 357-374. doi: $10.1017 / \mathrm{S} 0140525 \mathrm{X} 00058027$

[10] Kok, A. (2001) On the utility of P3 amplitude as a measure of processing capacity. Psychophysiology, 38, 557577. doi:10.1017/S0048577201990559

[11] Kutas, M., McCarthy, G. and Donchin, E. (1977) Augmenting mental chronometry: The P300 as a measure of stimulus evaluation time. Science, 197, 792-795. doi: $10.1126 /$ science. 887923

[12] Delpont, E., Dolisi, C., Suisse, G., Bodino, G. and Gastaud, M. (1991) Visual evoked potentials: Differences related to physical activity. International Journal of Sports Medicine, 12, 293-298. doi:10.1055/s-2007-1024684

[13] Ozmerdivenli, R., Bulut, S., Bayar, H., Karacabey, K., Ciloglu, F., Peker, I. and Tan, U. (2005) Effects of exercise on visual evoked potentials. International Journal of Neuroscience, 115, 1043-1050. doi:10.1080/00207450590898481

[14] Taddei, F., Viggiano, M.P. and Mecacci, L. (1991) Pattern reversal visual evoked potentials in fencers. International Journal of Psychophysiology, 11, 257-260. doi:10.1016/0167-8760(91)90019-T

[15] Hayashi, S., Hasegawa, Y., Yahagi, S. and Kasai, T. (2001) Modulation of motor evoked potentials induced by motor imagery: An analysis of trained and untrained Kendoists using a transcranial magnetic stimulation (TMS) method. Japan Journal of Physical Education,
Health and Sport Sciences, 46, 47-59.

[16] Caramia, M.D., Desiato, M.T., Cicinelli, P., Iani, C. and Rossini, P.M. (1993) Latency jump of "relaxed" versus "contracted" motor evoked potentials as a marker of cortico-spinal maturation. Electroencephalography and Clinical Neurophysiology, 89, 61-66. doi:10.1016/0168-5597(93)90086-5

[17] Syrjälä, P., Luukinen, H. and Tolonen, U. (2000) Motor evoked potentials of subjects over 70 years of age with and without recurrent falls. Clinical Neurophysiology, 111, 482-488. doi:10.1016/S1388-2457(99)00263-1

[18] Hoffman, L.D. and Polich, J. (1999) P300, handedness, and corpus callosal size: Gender, modality, and task. International Journal of Psychophysiology, 331, 163-174. doi:10.1016/S0167-8760(98)00050-6

[19] Numata, K., Nakajima, Y. and Shimizu, S. (1998) Right hemisphere dominance for figure recognition: A study of event related potentials to lateralized projection. Journal of the Japanese Physical Therapy Association, 25, 1-5.

[20] Astafiev, S.V., Shulman, G.L., Stanley, C.M., Snyder, A.Z., Van Essen, D.C. and Corbetta, M. (2003) Functional organization of human intraparietal and frontal cortex for attending, looking, and pointing. The Journal of Neuroscience, 23, 4689-4699.

[21] Callaway, E.M. (1998) Local circuits in primary visual cortex of the macaque monkey. Annual Review of Neuroscience, 21, 47-74. doi:10.1146/annurev.neuro.21.1.47

[22] Yoshimura, H., Mashiyama, Y., Kaneyama, K., Nagao, T. and Segami, N. (2007) Opening of shortcut circuits between visual and retrosplenial granular cortices of rats. Neuroreport, 18, 1315-1318. doi:10.1097/WNR.0b013e32827420fa

[23] Mehta, A.D., Ulbert, I. and Schroeder, C.E. (2000) Intermodal selective attention in monkeys. II: Physiological mechanisms of modulation. Cerebral Cortex, 10, 359-370. doi:10.1093/cercor/10.4.359

[24] Raiguel, S.E., Lagae, L., Gulyàs, B. and Orban, G.A. (1989) Response latencies of visual cells in macaque areas V1, V2 and V5. Brain Research, 493, 155-159. doi:10.1016/0006-8993(89)91010-X

EOG, electrooculogram;

TMS, transcranial magnetic stimulation;

V1, primary visual cortex;

$\mathrm{V} 2$, secondary visual cortex;

MT, middle temporal.

VMRT, visuo-motor related time;

MEP, motor evoked potentials;

P3, P300;

EEG, electroencephalograms;

VEP, visual evoked potentials; 